\title{
Новые данные по генезису корунда - синего сапфира Ильменогорского комплекса, Южный Урал: предварительные результаты LA-ICP-MS микро-примесного картирования
}

\author{
Сорокина Е.С. ${ }^{1}$, Аносова М.O. ${ }^{1}$, Рассомахин М.А. ${ }^{2,3}$ \\ ${ }^{1}$ Институт геохимии и аналитической химии им. В.И. Вернадского РАН (ГЕОХИ РАН), Москва, \\ elensorokina@mail.ru \\ ${ }^{2}$ Ильменский государственный заповедникУрОРАH, Mиасc,miha_rassomahin@mail.ru \\ ${ }^{3}$ Институт минералогии УрО РАН, Миасс
}

Аннотация. В статье приводятся предварительные результаты LA-ICP-MS микро-примесного картирования кристаллов зонального сине-коричневого корунда (сапфира) из сиенит-пегматитов жилы 298 Ильменогорского комплекса. Показано влияние содержания микропримеси железа на коричневую окраску минерала. Формирование зональных кристаллов корунда вероятно проходило в течение двух стадий минералообразования, зафиксированных в изменении его геохимических особенностей.

Ключевые слова: корунд, геохимия, LA-ICP-MS картирование, сиенит-пегматиты, Ильменогорский комплекс.

\section{New data on the genesis of corundum - blue sapphire of the Ilmenogorsky complex, South Urals: preliminary results of LA-ICP-MS trace-element mapping}

\author{
Sorokina E.S. ${ }^{1}$, Anosova M.O. ${ }^{1}$, Rassomakhin M.A. ${ }^{2,3}$ \\ ${ }^{1}$ Vernadsky Institute of Geochemistry and Analytical chemistry RAS (GEOKHI RAS), Moscow, \\ elensorokina@mail.ru \\ ${ }^{2}$ Ilmen State Reserve UrORAS, Miass, miha_rassomahin@mail.ru \\ ${ }^{3}$ Insitute of Mineralogy UrORAS, Miass
}

\begin{abstract}
The article presents preliminary results of LA-ICP-MS mapping of zonal blue-brown corundum (sapphire) crystals from syenite-pegmatite mine 298 in the Ilmenogorsky complex. The influence of increasing the iron trace element content on the brown color of the mineral is shown in the article. The formation of zonal corundum crystals likely occurred during the two stages of mineral formation recorded in a change in its geochemical features.

Key words: corundum, geochemistry, LA-ICP-MS mapping, syenite-pegmatites, Ilmenogorsky complex.
\end{abstract}

\section{Введение}

Корунд $\alpha-\mathrm{Al}_{2} \mathrm{O}_{3}$ является типичным минералом многих магматических и метаморфических пород. Однако его разновидность - синий сапфир, окрашенный ионами $\mathrm{Fe}^{3+}$ и/или $\mathrm{Fe}^{2+} / \mathrm{Ti}^{4+}$, встречается только в нескольких типах вмещающих пород, обогащенных глиноземом и обедненных кремнеземом. Между тем, производство сапфиров ювелирного качества сосредоточено главным образом во вторичных месторождениях дискуссионного генезиса (Sorokina et al., 2016, 2017). Следовательно, исследование генетической природы образования синего сапфира, обнаруженного in situ во вмещающих породах, может потенциально помочь в понимания петрогенеза корундов, обнаруженных на вторичных россыпных объектах.

В Ильменских горах на Южном Урале синий корунд обнаружен в сиенит-пегматитах жил 210, 298, 299, 311 и 349. Корундовые сиенит-пегматиты обнаружены в экзоконтактовой зоне основного интрузивного тела Ильменогорского комплекса, нефелиновых сиенитах (миаскитах), и генетически связаны с ними. Кристаллы корунда часто зональные по цвету (серо-синие или коричнево-синие) размером до 6 см. При этом центральная часть часто коричневого цвета с многочисленными твердофазовыми микровключениями сдвойникованных игл ильменита в виде «распада твердого раствора». Дополнительно в коричневых зонах наблюдаются псевдо-вторичные трехфазные флюидные включения, где жидкая фаза представлена $\mathrm{CO}_{2}$, а дочерняя фаза - диаспором (Sorokina et al., 2016, 2017). В синей зоне флюидные включения не обнаружены. 


\section{Результаты и обсуждение}

Нами были проведены первые эксперименты по разработке методики микро-примесного картирования при помощи масс-спектрометрии с индуктивно-связанной плазмой и лазерной абляцией (LA-ICP-MS) образцов зонального (сине-коричневого) корунда жилы 298 сиенит-пегматитов Ильменогорского комплекса (рис. 1). LA-ICP-MS является локальным микро-разрушающим методом, который активно используется в исследовании геологических объектов последние 40 лет. В сравнении с электронно-зондовым микроанализом, LA-ICP-MS имеет более низкий порог обнаружения для измерения различных элементов (до долей рpmw). Тогда как в отличие от масс-спектрометрии вторичных ионов (SIMS), LA-ICP-MS является более экспрессным методом и позволяет получать большие объемы данных за сравнительно короткий период времени. Измерения проведены после-

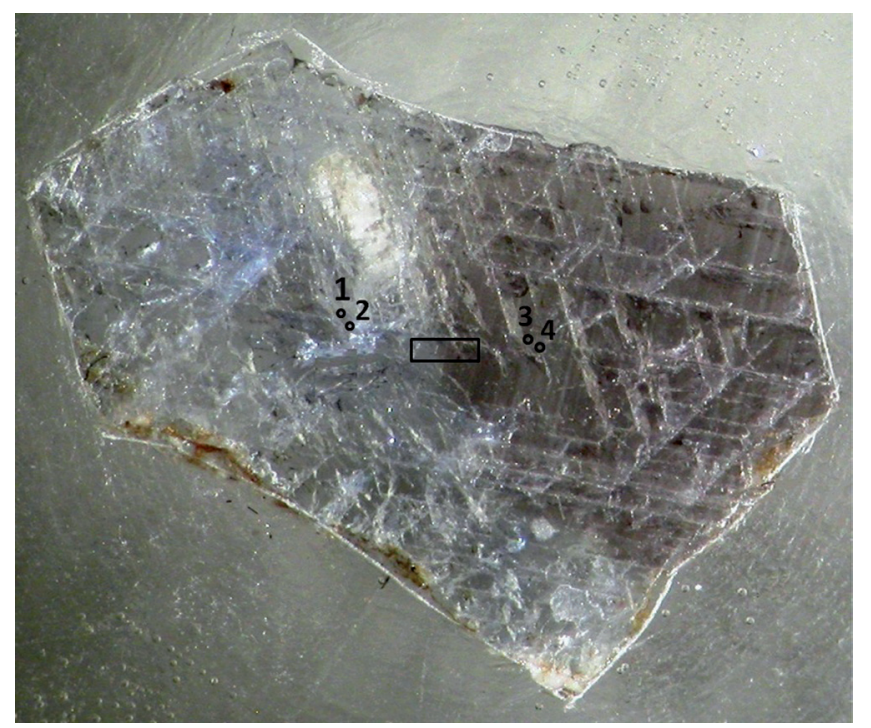

Рис. 1. Образец корунда размером $1.1 \times 0.6$ см из сиенит-пегматитов жилы 298, использованный для LA-ICP-MS картирования. Черным прямоугольником обозначена область измерения, черные круги - контрольные точки 1, 2, 3 и 4.

Fig. $1.1 .1 \times 0.6 \mathrm{~cm}$ corundum sample from syenitepegmatite mine 298 used for LA-ICP-MS mapping. The black rectangle is the measured area; the black circles are the reference points $1,2,3$ and 4 .

Таблица 1. LA-ICP-MS микро-примесный состав корунда (ppmw) из сиенит-пегматитов жилы 298 Ильменогорского комплекса, Южный Урал.

Table 1. LA-ICP-MS trace-element composition of corundum (ppmw) from syenite-pegmatite mine 298 of the Ilmenogorsky complex, South Urals.

\begin{tabular}{|c|c|c|c|c|c|c|c|c|c|c|c|}
\hline Элемент & Т 1* & T 2 & Т 3 & T 4 & P1 - 1 & P1 -2 & P1 -3 & P1 -4 & P1 - 5 & P1 -6 & P1 - 7 \\
\hline $\mathrm{Be}^{9}$ & bdl & bdl & 14 & bdl & bdl & bdl & bdl & bdl & bdl & bdl & bdl \\
\hline $\mathrm{Ca}^{44}$ & bdl & bdl & bdl & bdl & bdl & bdl & bdl & 397 & bdl & bdl & bdl \\
\hline $\mathrm{Ti}^{47}$ & bdl & 105 & 154 & 107 & bdl & 24 & 57 & 36 & 44 & 35 & 34 \\
\hline $\mathrm{V}^{51}$ & 12 & 13 & 15 & 16 & 13 & 13 & 15 & 15 & 16 & 15 & 16 \\
\hline $\mathrm{Mn}^{55}$ & bdl & bdl & bdl & bdl & bdl & bdl & 2,8 & 4.5 & bdl & 4 & bdl \\
\hline $\mathrm{Fe}^{57}$ & 3322 & 3631 & 5527 & 5802 & 3429 & 3616 & 3741 & 3891 & 3971 & 3916 & 4038 \\
\hline $\mathrm{Zn}^{66}$ & bdl & bdl & bdl & bdl & bdl & bdl & bdl & bdl & bdl & bdl & 10 \\
\hline $\mathrm{Ga}^{71}$ & 190 & 182 & 208 & 211 & 204 & 207 & 206 & 205 & 219 & 190 & 205 \\
\hline $\mathrm{Y}^{89}$ & bdl & bdl & bdl & bdl & 0,2 & bdl & 0.2 & bdl & bdl & bdl & bdl \\
\hline $\mathrm{Zr}^{91}$ & bdl & bdl & bdl & bdl & bdl & bdl & bdl & bdl & 7 & bdl & bdl \\
\hline $\mathrm{Nb}^{93}$ & bdl & 0.1 & bdl & bdl & bdl & bdl & 0.1 & bdl & 0.6 & 0,1 & bdl \\
\hline $\mathrm{Ta}^{181}$ & bdl & bdl & bdl & bdl & bdl & bdl & bdl & bdl & 0.7 & bdl & bdl \\
\hline $\mathrm{Pb}^{208}$ & bdl & 0.5 & 0.2 & bdl & bdl & bdl & bdl & bdl & 0.2 & bdl & bdl \\
\hline
\end{tabular}

Примечание. *T1, Т2, Т3, Т4 - контрольные точки 1, 2 в голубой зоне и 3,4 - в коричневой зоне кристалла корунда; P1 - 1 - ряд 1, точка 1 и т.д.; $\mathrm{Li}^{6}, \mathrm{Mg}^{24}, \mathrm{Sc}^{45}, \mathrm{Cr}^{53}, \mathrm{Co}^{59}, \mathrm{Ni}^{60}$ и $\mathrm{Sr}^{88}$ были измерены, но полученные значения были ниже порога обнаружения (bdl). Порог обнаружения для $\mathrm{Mn}^{55}-1.8-2.4$ ppmw, $\mathrm{Y}^{89}-0.1-0.2$ ppmw, $\mathrm{Nb}^{93}-0.05-0.1$ ppmw, $\mathrm{Ta}^{181}-0.05-0.1$ ppmw, $\mathrm{Pb}^{208}-0.1-0.3$ ppmw. 
Продолжение табл. 1

Table 1 contd.

\begin{tabular}{|c|c|c|c|c|c|c|c|c|c|c|c|c|c|c|c|c|c|c|}
\hline $\begin{array}{l}\text { D̃ } \\
\sum_{0}^{0} \\
\stackrel{0}{n}\end{array}$ & $\mathrm{P} 2-1 *$ & $\mathrm{P} 2-2$ & P2-3 & P2-4 & P2-5 & P2-6 & $P 2-7$ & P2-8 & P2-9 & P3-1 & P3-2 & P3-3 & P3-4 & P3-5 & P3-6 & P3-7 & P3-8 & P3- \\
\hline $\mathrm{Li}^{6}$ & & & & bdl & 46 & bdl & bdl & & & & bdl & bdl & bdl & (1) & 43 & & bdl & bdl \\
\hline $\mathrm{Mg}^{24}$ & bdl & bdl & bdl & bdl & bdl & bdl & bdl & bdl & bdl & bdl & 31 & bdl & bdl & bdl & bdl & bdl & bdl & bdl \\
\hline $\mathrm{Ca}^{44}$ & bdl & bdl & 339 & bdl & bdl & bdl & bdl & bdl & bdl & bdl & bdl & bdl & bdl & bdl & bdl & bdl & bdl & bdl \\
\hline $\mathrm{Ti}^{47}$ & 30 & 32 & 69 & bdl & 99 & 59 & 56 & bdl & 64 & 18 & 24 & 30 & bdl & 63 & 84 & 50 & 30 & 90 \\
\hline $\mathrm{V}^{51}$ & 14 & 14 & 14 & 14 & 15 & 15 & 17 & 16 & 15 & 14 & 14 & 12 & 16 & 14 & 16 & 16 & 14 & 15 \\
\hline $\mathrm{Cr}^{53}$ & bdl & bdl & bdl & bdl & 11 & bdl & bdl & bdl & bdl & bdl & 26 & bdl & bdl & bdl & bdl & bdl & bdl & bdl \\
\hline $\mathrm{Mn}^{55}$ & bdl & bdl & bdl & bdl & 7 & bdl & bdl & bdl & 2.76 & bdl & bdl & 3 & bdl & bdl & 3 & bdl & bdl & 3 \\
\hline $\mathrm{Fe}^{57}$ & 4485 & 4439 & 4561 & 4265 & 4649 & 4104 & 3997 & 4141 & 4196 & 4345 & 4407 & 4734 & 4487 & 4865 & 5036 & 4882 & 4931 & 5308 \\
\hline $\mathrm{Ga}^{71}$ & 194 & 210 & 203 & 205 & 207 & 201 & 208 & 203 & 219 & 210 & 198 & 205 & 208 & 195 & 206 & 202 & 204 & 197 \\
\hline $\mathrm{Sr}^{88}$ & bdl & bdl & bdl & bdl & bdl & bdl & bdl & bdl & bdl & bdl & bdl & 2 & bdl & bdl & bdl & bdl & bdl & bdl \\
\hline $\mathrm{Y}^{89}$ & bdl & bdl & bdl & bdl & bdl & bdl & bdl & bdl & bdl & bdl & bdl & bdl & bdl & bdl & bdl & 0.1 & bdl & bdl \\
\hline $\mathrm{Nb}^{93}$ & bdl & bdl & 0.2 & 0.1 & 0.1 & bdl & 0.1 & bdl & bdl & 0.2 & 0.1 & bdl & bdl & bdl & bdl & bdl & bdl & 0.1 \\
\hline $\mathrm{Ta}^{181}$ & bdl & bdl & 0.1 & bdl & bdl & bdl & & bdl & bdl & 0.1 & bdl & bdl & bdl & 0.1 & bdl & bdl & bdl & bdl \\
\hline $\mathrm{Pb}^{208}$ & bdl & bdl & bdl & bdl & bdl & 0.1 & bdl & 0.1 & bdl & 0.2 & 0.3 & bdl & bdl & bdl & bdl & bdl & bdl & 0.3 \\
\hline
\end{tabular}

Примечание. *P2 - 1 - ряд 2, точка 1 и т.д.; $\mathrm{Be}^{9}, \mathrm{Sc}^{45}, \mathrm{Co}^{59}, \mathrm{Ni}^{60}, \mathrm{Zn}^{66}$ и $\mathrm{Zr}^{91}$ были измерены, но полученные значения были ниже порога обнаружения; Порог обнаружения для $\mathrm{Sr}^{88}-1-2 \mathrm{ppmw}, \mathrm{Y}^{89}-0.1-0.2 \mathrm{ppmw}$, $\mathrm{Nb}^{93}-0.05-0.1$ ppmw, $\mathrm{Ta}^{181}-0.05-0.1$ ppmw, $\mathrm{Pb}^{208}-0.1-0.3$ ppmw.

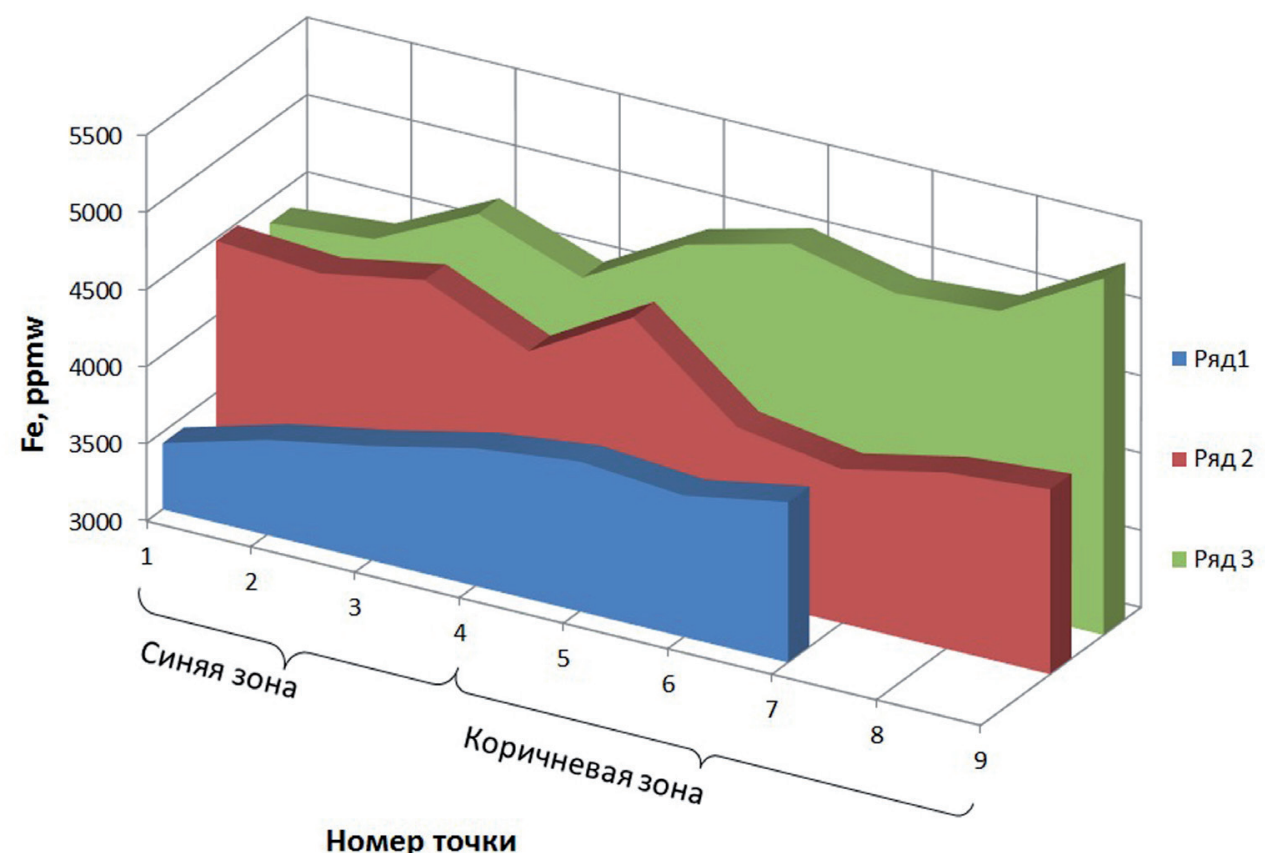

Рис. 2. Содержание примеси железа в разных цветовых зонах корунда сиенит-пегматитов жилы 298 по данным LA-ICP-MS картирования.

Fig. 2. The content of iron trace element in differently colored zones of the corundum from syenite-pegmatite mine 298, according to LA-ICP-MS mapping data. 
довательно в точках диаметром 30 мкм, расположенных в виде сетки с шагом 5 мкм в переходной цветовой зоне образца. Частота измерения $10 \mathrm{~Hz}$, энергия 14 Дж/см² с измерением в течении $60 \mathrm{c}$, wash in/wash out - 80 с и 90 с. Подобраны оптимальные стандарты - искусственные стекла NIST 610, и NIST 612, а также природное стекло ATHO-G было использовано для контроля качества анализов (QCM); стандарты и QMC измеряются блоками через каждые 2 часа съемки. Ошибка измерения стандартов и QCM не превышала 10-15 \% от предпочтительных значений элементов, бо́льшая ошибка измерения связана с интерференцией элементов, которую нельзя избежать при использовании данного метода.

Было измерено 3 ряда точек (см. табл. 1) и 4 контрольные точки в двух цветовых зонах образца (по 2 точки на зону). Полученные значения содержания микропримесей $\mathrm{Ti}, \mathrm{Fe}, \mathrm{V}$ и $\mathrm{Ga}$ в корунде примерно одинаковые по сравнению теми, полученными ранее при использовании этого же метода, а также с помощью электронно-зондового микроанализа (Sorokina et al., 2016, 2017). Таким образом установлено, что коричневая зона образца более обогащена железом по сравнению с синей зоной (на рис. 2 видно осцилляционное накопление примеси железа в коричневой зоне кристалла). При этом во втором ряду точек, напротив, видно снижение содержания железа в коричневой зоне (см. рис. 2), что, вероятно, связано с захватом микровключений ильменита с синей зоне (в точках 3-5 и 7-2-го ряда видно накопление примесей $\mathrm{Nb}$ и Та, нехарактерных для корунда). Таким образом, полученные данные согласуются с данными UV-Vis-NIR спектроскопии: окраска корундов связана преимущественно с ионами $\mathrm{Fe}^{3+}$; в спектре корундов не наблюдались пики обменно-связанных пар ионов $\mathrm{Fe}^{2+}-\mathrm{Ti}^{4+}$ (Sorokina et al., 2017). Диагностика флюидных включений только в коричневой зоне кристаллов корунда связана скорее всего с перерывом в минералообразовании и формировании синей зоны кристалла позднее при последующих термальных событиях, что согласуется с данными геохронологии (неопубликованные данные Сорокиной Е.С.).

Работа выполнена при поддержке гранта Президента Российской Федерации (№ проекта МК4459.2018.5).

\section{Литература}

1. Sorokina E.S., Koivula J.I., Muyal J., Karampelas S., Nishanbaev T.P., Nikandrov S.N. Multiphase fluid inclusions in blue sapphires from the Ilmen Mountains, Southern Urals // Gems and Gemology. 2016. V. 52 (2). P. 209-211.

2. Sorokina E.S., Karampelas S., Nishanbaev T.P., Nikandrov S.N., Semiannikov B.S. Sapphire megacrysts in syenite pegmatites from the Ilmen Mountains, south Urals, Russia: New mineralogical data // Canadian Mineralogist. 2017. V. 55 (5). P. 823-843. 